\title{
A New Perspective on Time and Physical Laws
}

\author{
Lucy James \\ University of Bristol
}

September 29, 2020

\section{Acknowledgements}

This work was made possible by numerous extensive conversations with many people. Special thanks go to Karim Thébault, Sam Fletcher, Pete Evans, Henrique Gomes, James Ladyman and Rasmus Jaksland for invaluable correspondence and comments on earlier drafts. Thanks also to Craig Callender, Pete Evans and Sam Baron, whose work provided the initial inspiration to pursue the topics written about here. Finally, thanks to the referees for their constructive comments on an earlier version.

\begin{abstract}
Callender claims that 'time is the great informer' (Callender 2017, chapter 7), meaning that the direction(s) in which our 'best' physical theories inform are temporal. This is intended to be a metaphysical claim, and as such expresses a relationship between the physical world and information-gathering systems such as ourselves. This paper gives two counterexamples to this claim, illustrating the fact that time and informative strength doubly dissociate, so the claim cannot be about physical theories in general. The first is a case where physical theories inform in directions that we have no reason to regard as temporal. The second is a case where our best physical theories fail to inform in directions that we have independent (pre-theoretic) reasons to regard as temporal. Taking these two cases into account suggests that the connection Callender makes between time and informativeness is perspectival. The second case demonstrates that, although scientists often seek
\end{abstract}


information in temporal directions, the behaviour of the physical world can present serious difficulties for finding it. In response, this paper proposes a perspectival reading of Callender's claim, according to which the connection between time and informative strength has more to do with the aims and objectives of science than the workings of the physical world.

\section{Introduction}

We begin with the problem of how to distinguish time from space in post-relativistic physics. It will be clear to many readers that time and space are essentially different, at least in the way we experience them. Getting lost, for example, is a rather different problem to being late; travel is permitted in any direction of space, but the same cannot be said for time; and, spatially distant objects can be seen or accessed in a way that temporally distant objects cannot. These (and other) differences are encoded in a host of ways across physics. Despite relativistic physics teaching us about some important inter-dependence relationships between space and time, their differences are nonetheless encoded by asymmetries in the models of the theory. There remain questions about whether there might be a philosophical underpinning of the various asymmetries between time and space that we find in physical theories, and about whether such asymmetries are physically necessary. ${ }^{1}$

Callender addresses these questions with his attempt to 'bind together' the differences between time and space, by claiming that 'time is the great informer' (Callender 2017, chapter 7), meaning that the direction in which the laws of our 'best' theories inform are temporal, not spatial. $^{2}$ This paper gives two counterexamples to this claim. The first is a case where physical theories inform in directions that we have no reason to regard as temporal. The second is a

\footnotetext{
${ }^{1}$ To be clear, this paper is about asymmetries between time and space, and not about the asymmetry of time. It has nothing to do with differences between past and future for example, and the thermodynamic arrow of time will not be mentioned. It is about differences between time and space, and to the extent that spacetime can be thought of as a single structure it is appropriate to refer to the 'asymmetry' of this structure as encoding some of these differences.

${ }^{2} \mathrm{~A}$ different response might consider this asymmetry to be a defining characteristic of any structure that can properly be called spatiotemporal. A proposal of this nature has been made use of in Le Bihan and Linnemann 2019, in order to strengthen connections between the asymmetric spacetime of general relativity and asymmetric 'quasi-spacetime' structures in theories of quantum gravity.
} 
case where our best physical theories fail to give precise information over short timescales or accurate information over long timescales, where we have independent (pre-theoretic) reasons to regard these directions as temporal. Taking these two cases into account breaks the connection Callender makes between time and informative strength of physical laws. The second case demonstrates that, although scientists often seek information in temporal directions, the behaviour of the physical world can present serious difficulties for finding it. This motivates the view that the connection between time and informative strength has more to do with the aims and objectives of science than the workings of the physical world.

The positive proposal of this paper, namely that time is connected to the informative strength of physical laws only for a certain set of scientific aims, is related to 'temporal perspectivalism', a position advanced in Baron and Evans forthcoming. Their reading of Callender's argument leads to the conclusion that the asymmetry between time and space is an artefact of our human epistemic situation. The alternative interpretation offered in the present work is also a form of perspectivalism, but the scope is narrower: it is only the connection Callender makes between time and informative strength, and not the asymmetry between time and space per se, that is reduced to a set of perspectives. However, the persepctivalism in this case is more worrying for objectivity, because the set of perspectives in question is identified by the specific aims of some human scientists, rather than the epistemic situation of the entire population. The proposal is rooted in empiricism (which Callender claims also to be committed to), a weak version of which can be spelt out as follows: the informativeness of physical laws is contingent on their formal characteristics being a good representation of empirically accessible phenomena.

The structure of this paper is as follows. Section 2 gives a summary of the main arguments including the 'perspectival' reading of Callender's argument offered in Baron and Evans forthcoming. Section 3 sets out the technical material necessary for understanding Callender's thesis and the two additional cases. Section 4 argues that the two additional cases are indeed counterexamples to Callender's claim, and explains how they motivate the alternative proposal 
connecting time to the aims of many scientific endeavours to provide certain forms of information. Subsection 4.3 compares the two forms of perspectivalism, gesturing towards some possible implications of their combination. Finally, section 5 briefly concludes.

\section{Overview}

Subsection 2.1 summarises the connection Callender makes between time and informativeness in physical laws. This is followed by an overview, in subsection 2.2 , of the reading of his arguments suggested by Baron and Evans, leading to their proposal for a perspectival take on the timespace asymmetry. It then outlines some further discussion of Callender's argument in 2.3, laying the groundwork for understanding the significance of the two counterexamples. Finally, also in 2.3, it outlines an alternative proposal: the connection between time and informativeness is an artefact of the aims and interests of scientists working on specific research programs. This alternative proposal can be viewed as a perspectival reading of Callender's argument, but where the perspectivalism applies specifically to the connection between time and informativeness rather than the asymmetry between time and space itself. This reading is, however, more problematic for the generality of Callender's metaphysical thesis than that suggested by Baron and Evans, because the perspectives to which his claim is reduced form a subset of the regimes used in scientific practice rather than the epistemic perspective of human beings in general.

\subsection{Time as the Great Informer}

Callender identifies features of time as it appears in physical theories, which distinguish it from space. He attempts to weave these together into a general metaphysical thesis by connecting to a Lewisian 'Best Systems Account' (henceforth, the BSA) of physical modality. ${ }^{3}$ His strategy is to use the principles of the BSA to provide criteria for identifying a representative set of physical laws, whose mathematical form he analyses in order to give a general message about spatio-temporal structure. His conclusion is that 'time is the great informer' - time is the set of directions on a four-dimensional manifold of events in which laws give the most information.

\footnotetext{
${ }^{3}$ See Lewis 1983 for details of this account. See also Massimi 2018a, Cohen and Callender 2009, Maudlin 2007, Loewer 1996, and Belot 2011 (to name but a few) for discussion.
} 
He summarises this view with the statement that, "time is that direction on the manifold of events in which we can tell the strongest or most informative stories.' (Callender 2017, p.142). So, the most informative directions on the manifold are labelled as 'time'. We begin with the notion of informativeness and end up with a set of privileged 'timelike' directions. I call this hypothesis Callender's Temporal Informativeness Proposal, and refer to it hereafter as TIP.

Callender gives several slight variants of this overall conclusion. In chapter 6 he writes, 'the temporal direction is that direction on the manifold of events in which our best theories can tell the strongest, most informative "stories". Put another way, time is that direction in which our theories can obtain as much determinism as possible.' (Callender 2017, p.120) A slightly more precise formulation is given in chapter 7: 'A temporal direction at a point $p$ on $\left\langle M^{d}, g\right\rangle$ is that direction $(n,-n)$, where $(n,-n)$ is an unordered pair of nowhere vanishing vectors, in which our best theory tells the strongest, i.e., most informative, "story".' His strategy does not "assume that the "timelike" directions of $g$, if any, are themselves temporal directions. That's something we hope emerges from the analysis, not something put in.' (Ibid, p.142/143).

By way of explanation for his proposed conclusion, Callender states that, 'strength is linked to time because it is deeply connected to the other temporal features of our universe. If I am right, "strength" is the glue that binds together many otherwise detachable features central to time.' (Ibid, p.120). This paper does not aim to outright deny that there is any link at all between strength (informativeness) and time, but instead to propose a different, perspectival, explanation for it. It does so by acknowledging the lack of generality of the link Callender makes between strength (informativeness) and time, questioning what it is that unites the examples in physics where the link obtains and comparing them to examples where this link does not obtain. Callender's claim is that his 'attractive idea about time is more or less implied by a "systems" approach to laws'. He states also that 'the difference [between time and space, based on the way laws inform] ultimately lay in the distribution of physical properties' (p.156). This part of his thesis will also be challenged, especially through the analysis of chaotic systems in subsection 3.3. 
The argument for TIP is explicitly based on a Humean view of laws, according to which 'laws are simply the best summary of the facts' (ibid, p.140). This is where a commitment to Humean metaphysics and empiricist epistemology is made. According to Callender (p.140), 'Humean theories seek to explain the laws given the distribution of actual facts', the actual facts being facts which are (at least in principle) empirically accessible. ${ }^{4}$ In the language of David Lewis, the laws supervene on the mosaic of particular facts (see Lewis 1983), meaning that there could not be a change in the laws without there being a corresponding change in the particular facts they describe (Bennett and McLaughlin 2005). There are formally different ways to describe the same mosaic of facts, but these descriptions will not differ in content. The BSA provides a way of distinguishing between these formally different descriptions, elevating to the status of law parts of those descriptions, taking the form of deductive systems, which satisfy some set of 'theoretical virtues', in particular simplicity and strength. ${ }^{5}$ Callender invites us to 'consider various deductive systems, each of which only makes true claims about what exists' (p.140). We then employ the theoretical virtues of strength and simplicity, emphasising strength in particular, to identify the systems and associated laws which give the best description of the world. 'The motivation for the [BSA] theory is', according to (Callender 2017 p.140), 'the idea that physical laws seek to describe accurately as much of the world as possible in a compact way. ${ }^{6}$ TIP comes out of an analysis of laws which, Callender claims, achieve this goal.

We need not concern ourselves very much with the notion of simplicity. Callender briefly discusses the simplifying role that time plays in physical laws, but asserts that 'There is nothing special about time here. [...] Space, for instance, is also the great simplifier.' For this reason, we must focus also on the virtue of strength, thought of as informativeness. 'In balancing simplicity and strength, a best system will [...] contain a way to generate some pieces of the domain of events given other pieces. In other words, it will favour algorithms, and short ones at

\footnotetext{
${ }^{4}$ Taking the distribution of empirical facts as given follows from the commitment to empiricism, central to Humean theories in general.

${ }^{5}$ Theoretical virtues may also include uniformity, elegance, generality and perhaps more, but we follow Callender in focusing only on simplicity and strength.

${ }^{6}$ Seeking to describe the world is another expression of the empiricist commitments of Humean theories, if 'the world' is taken to refer to that which is observable, measurable or otherwise empirically accessible. If 'the world' consisted of more than this it would be difficult to understand laws as being descriptive, because we would have no way of comparing descriptions with what they describe.
} 
that. The more of what happens that is generated by small input the better.' (Callender 2017, p.141; my emphasis). The claim is that a privileged time parameter is what gives physical laws their informative strength.

There are two versions of this argument given, an 'informal' one in chapter 7 and a 'formal' one in chapter 8 . It is indicated that these two versions of the argument are supposed to work together, that the latter version is intended to flesh out the former, adding credibility to it with the investigation into formal characteristics of equations used in physics. This version of his argument in chapter 8 proceeds by identifying a broad set of physical laws, generalised by their mathematical form, which provide algorithms that are informative in this sense. These laws take the form of partial differential equations (PDEs) which support well posed Cauchy problems. He investigates the mathematical form of these PDEs, and it is in this mathematical form that we find a privileged set of 'informative' directions that are defined as 'timelike': these equations use antecedent data to provide maximal information in timelike directions.

PDEs which support Cauchy problems do provide concise algorithms and are particularly informative over their domains of applicability. Section 4 explores Callender's justification for singling out these laws as being maximally informative, regardless of applicability. As described in section 3, it is their geometric structure, specifically, they are hyperbolic, that makes the distinction between timelike and spacelike directions. Hyperbolic PDEs, when defined on some manifold, divide the tangent space of each point of that manifold into three regions by a pair of intersecting cone-shaped surfaces, in a manner analogous to the light-cone structure of special relativity and locally of general relativity. Restricting to well-posed problems means that antecedent data can only be defined on surfaces corresponding to spacelike regions, and the PDEs evolve this in directions that correspond to the timelike. Therefore, the physical laws selected using the BSA criteria distinguish time from space, where the timelike and not the spacelike directions are informative.

Callender suggests two alternative ways to read his proposal: one 'conservative' and one 
'radical'. So far we have been vague about what exactly the mosaic of facts or manifold of events or supervenience basis consists of. The two readings of the argument, to some degree, take care of this issue. According to the more conservative reading, the supervenience basis consists of events on a Lorentzian manifold endowed with a spacetime metric, which has an asymmetry between time and space built into its signature. The best systems merely embody the structure already assumed to be present in the manifold; the distinction between time and space does not emerge through systematisation, but the connection between time and informativeness does. According to Callender, however, the 'radical perspective is the more natural development of the theory.' (Callender 2017, p.151). This second reading assumes only 'the events $e$ on $M$ ' and the best systems 'arrive at the spacetime metric $g$ and laws $L$ together' (Callender 2017, p.150). So, Callender's preferred interpretation of TIP means that, by focusing on informative laws, the asymmetry between time and space is derived through systematisation, and time is defined as the set of directions that are maximally informative.

\subsection{Temporal Perspectivalism}

Samuel Baron and Peter Evans (in Baron and Evans forthcoming) explore some consequences of adopting the radical reading of TIP, according to which, 'the metrical difference between the timelike and spacelike - the centerpiece of all our physical theories - also [in addition to the link between time and informativeness] depends on the system' (Callender 2017, p.151, emphasis in original). What they propose is a thesis they call 'temporal perspectivalism', where the distinction between time and space is only objective "so long as this is understood in a deflationary, epistemic sense.' (Baron and Evans forthcoming). ${ }^{7}$ They admit some invariant structure in the world, existing independently of any perspective, which we then divide into timelike and spacelike directions 'based on our idiosyncratic epistemic constraints and limitations concerning that structure' (Baron and Evans forthcoming, p.18), so temporal directions are picked out both by the notion of informativeness and the epistemic perspectives of agents, and are not part of the invariant structure. To be clear, they do not refute TIP:

\footnotetext{
${ }^{7}$ Their view is inspired by, and analogous to, the 'causal perspectivalism' of Huw Price and Jenann Ismael (see Price and Corry 2007 and Ismael 2016). This should not be confused with 'perspectival realism' (see Massimi 2016, Giere 2010 and Teller 2011, for example).
} 
they do not contest that the directions of maximal informativeness in our laws pick out time. Instead, the resulting asymmetry between time and space is considered to be a mere artefact of our human perspective, and this view is a consequence of accepting the radical version of TIP. ${ }^{8}$

To flesh this out, as a thought experiment, they consider the possibility of Martian scientists who do not share our epistemic situation within the invariant structure of the world. ${ }^{9}$ Imagine that these Martians are 'smeared out' across what we call the temporal dimension and two of our spatial dimensions, and they seek laws that inform along their forth dimension (which would be spatial, for us). They would identify their own natural kinds, as we do, according to the kinds of empirical data available to them, and 'one could imagine complete incomensurability between the two ways of carving up the world' (Baron and Evans forthcoming). Hypothetically, they might design the same PDEs as us, based on the same concerns about informativeness, but would disagree about which directions on the manifold should be regarded as timelike. The point of temporal perspectivalism is that this would be a 'no-fault' disagreement: there is no fact of the matter about who would be correct. Even if communication with these Martians were possible, there would be no clear method for deciding which description to prioritise, and no reason to privilege one over the other in a perspective-independent way. We would have our 'time' as the directions in which our laws are maximally informative; they would have theirs. The 'mere possibility' of such Martian scientists, according to Baron and Evans, 'suggests that the distinction [between time and space] is pragmatic in origin.' ${ }^{10}$ (Baron and Evans forthcoming, p.16).

Their reasons for adopting this odd-sounding view come from taking the radical reading of Callender's arguments at face value. If time is defined as the direction in which well posed Cauchy problems are maximally informative, then we are at liberty to hypothesise agents for

\footnotetext{
${ }^{8}$ This consequence may be unpalatable to some readers, providing further motivation for looking for ways to reject $T I P$.

${ }^{9}$ To be clear, this Martian thought experiment is Callender's, but is described very clearly in Baron and Evans forthcoming and used there to elucidate their position.

${ }^{10}$ Note that pragmatic concerns can come from two sources: our epistemic situation, which is the focus of Baron and Evans, and particular aims and interests, which will be our focus later on. Of course these two sources are related.
} 
whom this direction turns out to be different from our own. After all, scientific laws are designed very much with our epistemic vantage point in mind - their very purpose is to give information about what we do not have direct empirical access to. For us, this is what we call the temporal future. For the Martians, this might be what we call East or West perhaps. Looking more closely at this thought experiment reveals that it is the orientation of the distinction between time and space that is discussed, not the existence of a distinction in the first place. 'Time' and 'space' are still distinguished for the Martians, only their 'time' is different to ours. We are not invited to imagine the laws that scientists from Venus might come up with if, for example, they had epistemic access to all four dimensions of the manifold. In this case, there would likely be 'no-fault' disagreements with us earthlings about whether there needs to be a privileged time dimension at all, if our concerns lie solely with informativeness. So for Baron and Evans it is taken to follow from Callender's arguments that there are privileged time-directions, but there may be disagreements between agents with differing perspectives about which directions these are. A similar thought experiment, combined with Callender's arguments, could lead to the very definition of time being a matter of perspective.

In what sense are the hypothetical Martian scientists possible? Clearly, their possibility cannot be a symptom of our physical laws because this would induce a circularity in the reasoning. Without an answer to this question, it is difficult to understand how we could motivate anything other than agnosticism about the objectivity of the directions we regard as temporal. Ours might be the only possible epistemic situation for cognitive agents - who knows? It is also difficult to see how any single scientific fact could avoid falling prey to this sort of perspectival reading, since science is necessarily built up from our epistemic perspective and makes use of empirical data we have access to. To avoid these difficulties, we shall explore the extent to which Callender's arguments induce a different sort of perspectival reading. Might there be alternative human perspectives which would disagree about which directions are temporal? If we define time as the direction in which laws are maximally informative, as has been suggested by both Callender and Baron and Evans, this indeed turns out to be the case. This is the case, so long as we do not restrict attention to well posed Cauchy problems. The 
disagreements between these different human perspectives would be based on their pragmatic concerns relating to the kinds of projects they engage in, since not all scientists specialise in dynamics. This points to an absurdity in defining time in this way; at best, informativeness can be an ingredient in a pluralistic definition of time.

\subsection{Temporal Aims}

We need not consider imaginary alien scientists who disagree about which directions are temporal in order to give a perspectival reading of Callender's thesis. Instead, we shall think about real human scientists whose laws inform in directions which are not regarded as temporal, and others who struggle to generate any accurate information in directions which are uniformly regarded as time by those scientists. TIP is reduced to a particular subset of human interests and pragmatic concerns. Unlike with the thought experiments of Baron and Evans, everyone agrees about which directions to regard as temporal. The 'no-fault' disagreements are about which directions are maximally informative, because this depends on what we wish to be informed about. The connection between time and informativeness is thus more strongly perspectival, because it is as much about the interests and aims of particular groups of scientists as the epistemic situation of humans, and so we replace TIP with TAP: Temporal Aims Proposal. ${ }^{11}$

There are several ingredients of Callender's argument that can be used to undercut TIP, one of which leads towards TAP. Our focus will be on the commitment to empiricism which, if we recall, is a fundamental ingredient of Humean theories, and Callender's proposal purports to be both Humean and empiricist. This commitment sits uncomfortably with Callender's method of conducting an analysis of the purely formal characteristics of laws as a way of generating metaphysical claims. If we perform a similar analysis whilst holding on to some basic empiricist principles, we arrive at the conclusion that time is not 'the direction on the

\footnotetext{
${ }^{11} \mathrm{My}$ analysis of TIP means that it can be thought of as a perspectival truth in Michaela Massimi's sense (see Massimi 2018b), because its truth is dependent on a particular set of scientific perspectives (where alternatives are available) as opposed to being dependent on the epistemic situation of an entire species.
} 
manifold in which we can tell our strongest or most informative stories' (Callender 2017, p.142; my emphasis), but is instead the direction in which we would most like to be able to tell our best stories. ${ }^{12}$ The applicability of the laws to observable, measurable or otherwise empirically accessible physical contexts is all-important here. My view is compatible with that of Baron and Evans but, unlike theirs, does not rely on adopting the so-called 'radical' reading of Callender's proposal; it follows from the 'conservative' reading too. For this reason, it is neither the distinction between time and space in general, nor the particular set of directions that are defined to be timelike, that are relativised to human perspectives. Instead it is only the specific connection between time and informativeness, and this is relativised to some human perspectives.

My main objection is almost pre-empted by Callender himself. 'Confining attention to the marks of strength but not to strength itself would be a mistake', he warns us. He then goes on to make this very mistake, and the rest of his argument follows from it. His analysis focuses on too narrow a set of laws (identified by the marks of strength they exhibit) to draw a general conclusion about the role of time. He goes on: 'The degree to which a theory is informative is determined by how much of the world it manages to imply, not (in the general theory, at least) by formal characteristics.' (Callender 2017, p.143; my emphasis). Attributing epistemic priority to the world over formal characteristics of theories is part of the commitment to empiricism, which is central to the BSA and to Humean metaphysics in general. ${ }^{13}$ Callender's subsequent analysis of Cauchy problems is in terms of their formal characteristics, therefore only managing to achieve an assessment of their hypothetical informativeness. Their actual informativeness is contingent on these formal characteristics being a good representation of the world. What we shall see is that there are many empirically accessible physical phenomena to which Cauchy problems simply do not apply. Perhaps more worryingly, the directions that are uniformly labelled as time are especially uninformative for many of these phenomena. Section 3 presents two examples to illustrate these points.

\footnotetext{
${ }^{12}$ The point here is to emphasise not only our pragmatic concerns that stem from our particular epistemic situation, but also those which are about particular interests.

${ }^{13}$ See for instance Lewis 1999, Gupta 2006, Cottingham 1988, Quine 1951, Carnap 1991 and Feyerabend 1965 for discussion of these issues.
} 
Ongoing research in the area of dynamical systems theory presented in section 3.3 is focused on developing techniques to try to make better predictions about the temporal future, but often these techniques are complex - thus requiring some relaxation of the other 'horn' of the BSA, namely simplicity. Nonetheless, one of the central aims of science is to make informative predictions over directions that we have independent (pre-theoretic) reasons to regard as temporal. The connection between time and informative strength in physical laws is thus more plausibly thought of as being entirely pragmatic and not metaphysical, reducible to the aims and interests of scientists rather than to the way the world is.

\section{Physical Laws}

This section sets out the technical material that is relevant to the arguments presented. In order to fully appreciate the central claims of this paper, it is necessary to engage with some mathematical details. Only those equations which contribute towards understanding the philosophical arguments are presented, and their relevance is explained. Section 3.1 describes the structure of PDEs which support well posed Cauchy problems, required to understand the details of Callender's proposal and the 'perspectival' interpretation of Baron and Evans. The mathematical details of two counterexamples to TIP, which instead motivate TAP, are given in sections 3.2 and 3.3. The first of these is a problem for both readings of TIP, while the second is problematic only for the radical interpretation. 


\subsection{Time and Informative Strength}

Cauchy problems are a kind of boundary value problem (BVP) which can be set for second order linear PDEs in $m$ independent variables. ${ }^{14}$ In its most general form:

$$
\phi\left(u, x_{i}, p_{i}, r_{i}, s_{i k}\right)=0
$$

for $i=1, \ldots, m ; k=1, \ldots, m$ for $i \neq k$. The solution of the equation, $u$, is our unknown function of the $m$ independent variables, $x_{i}$. The $x_{i}$ s represent physical quantities, and the solution $u$ describes a function relating these to one another. $\phi$ is some arbitrary function of the variables in parenthesis, where $p_{i}=\frac{\partial u}{\partial x_{i}}, r_{i}=\frac{\partial^{2} u}{\partial x_{i}{ }^{2}}$ and $s_{i k}=\frac{\partial^{2} u}{\partial x_{i} \partial x_{k}}$. The PDE is solved subject to antecedent conditions:

$A C_{1}: u\left(x_{1}, \ldots, x_{m-1}, 0\right)=u_{0}\left(x_{1}, \ldots, x_{m-1}\right)$,

$A C_{2}: \frac{\partial u}{\partial x_{m}}\left(x_{1}, \ldots, x_{m-1}, 0\right)=u_{1}\left(x_{1}, \ldots, x_{m-1}\right)$.

Antecedent data of this form is called Cauchy data, defining the problem as a Cauchy problem. Notice that both conditions $A C_{1}$ and $A C_{2}$ are known functions of $m-1$ of the $m$ independent variables, and so must be given on a hypersurface of dimension one less than the full solution space.

In order to be 'well posed', a Cauchy problem must satisfy the following three criteria:

$W P_{1}$ : A solution must exist.

$W P_{2}$ : The solution must be unique.

$W P_{3}$ : Solutions must vary continuously with antecedent data.

The first two of these criteria are jointly sufficient to define the equation as deterministic. ${ }^{15}$ They ensure that one and only one solution corresponds to each set of antecedent conditions.

\footnotetext{
${ }^{14} \mathrm{My}$ presentation of Cauchy problems and of PDEs is taken primarily from Hadamard 2003, Garabedian 1964, Klainerman 2010, I. Rubinstein and L. Rubinstein 1998, and Robinson 1998. The simplest Cauchy problem is set for a first order ordinary differential equation, and the definition of a Cauchy problem generalises to PDEs of order $n$. For ease of exposition, our discussion focuses only on the case of second order PDEs, since these are the most prevalent in physical problems.

${ }^{15}$ Note that this is a mathematical definition of a deterministic equation, and should not be confused with philosophical issues about deterministic theories or worlds.
} 
The third criterion is stronger, requiring that variations of the antecedent data map to corresponding variations of the solution by a continuous function. In practical applications this means that errors made in the computation of either $A C_{1}$ or $A C_{2}$ are not amplified, or at least that the amplification of errors can be controlled.

In order to establish conditions under which equation (1), together with antecedent conditions $A C_{1}$ and $A C_{2}$, meets the three criteria to be well posed, leading to the discussion of space and time, we express (1) in linear form:

$$
\sum_{i, k} A_{i k} \frac{\partial^{2} u}{\partial x_{i} \partial x_{k}}+\sum_{i} B_{i} \frac{\partial u}{\partial x_{i}}+C u=f
$$

where $A_{i k}$ is a matrix, $B_{i}$ is a vector and $C$ is a scalar, all of which we assume to be constant, and $f$ is some linear function of the independent quantities $x_{i} \cdot{ }^{16}$ Equation (2) may be classified according to the eigenvalues of $A_{i k}$ as follows:

- Elliptic iff all eigenvalues of $A_{i k}$ are non-zero and have the same sign;

- Hyperbolic iff all eigenvalues of $A_{i k}$ are non-zero, and all but one have the same sign;

- Parabolic iff any eigenvalues of $A_{i k}$ vanish.

Cauchy conditions $A C_{1}$ and $A C_{2}$ yield unique solutions only for hyperbolic PDEs. Other kinds of boundary condition (for example Dirichlet or Neumann boundary conditions, which will be defined in section 3.2) are either too restrictive for a solution to exist, or are not sufficient to give a unique solution. Conversely, Cauchy data is either too restrictive or insufficient to yield unique solutions for elliptic and parabolic equations. These require either Dirichlet or Neumann boundary conditions. Therefore, a well posed Cauchy problem consists of a hyperbolic PDE subject to conditions $A C_{1}$ and $A C_{2}$.

\footnotetext{
${ }^{16}$ We assume that equation (2) has constant coefficients in the interest of simplicity of presentation. In full generality, the coefficients of a second order linear PDE may be known functions of the $x_{i}$ s, but classification of PDEs with variable coefficients is much more difficult, though not impossible. For the sake of argument, we may assume that the results suitably generalise.
} 
The characteristics of a PDE place restrictions on where antecedent data can be defined in order to ensure the existence of a unique solution. ${ }^{17}$ Characteristics of equation (2) are defined to be surfaces or hypersurfaces $\xi\left(x_{1}, \ldots, x_{m}\right)=c$, for constant $c$, where $\xi$ is a solution of

$$
\sum_{i, k} A_{i k} \frac{\partial \xi}{\partial x_{i}} \frac{\partial \xi}{\partial x_{k}}=0
$$

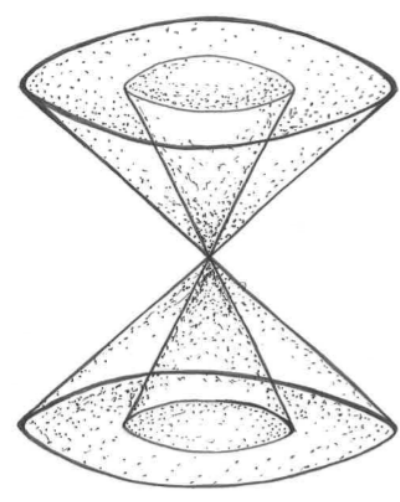

Figure 1: Conoid characteristic surfaces in $\mathbb{R}^{3}$. The width of the cones is set by the value of the constant c. For a continuous problem there are infinitely many of these surfaces, with a vertex at each point of the manifold.

Solving equation (3) for our different classes of PDE shows that elliptic PDEs have no real characteristics, and parabolic PDEs have one family of plane characteristics for each constant $x_{m}$. Hyperbolic PDEs, where Cauchy problems are well defined, have two families of conoid characteristics. In $\mathbb{R}^{3}$ these are pairs of two-dimensional cone-shaped surfaces sharing a vertex at each point of the domain. In higher dimensional spaces, conoid characteristics are higher dimensional generalisations of this basic structure. Figure 1 shows a geometric representation of the characteristics of a hyperbolic PDE in $\mathbb{R}^{3}$.

The Cauchy-Kowalewski theorem states that, to ensure the existence of a unique solution, antecedent data for a PDE must not intersect or be tangent anywhere to a characteristic. ${ }^{18}$ Recall that antecedent conditions $A C_{1}$ and $A C_{2}$ must be defined on a (hyper)surface of dimension one less than the full solution space, and consider where such a surface may be placed so as to avoid the characteristics of a hyperbolic PDE. As shown in figure 2 , in $\mathbb{R}^{3}$ this

\footnotetext{
${ }^{17}$ Characteristics are important for several other reasons too, for example in many cases solving the 'characteristic equation' associated with a PDE is a crucial step in solving the PDE itself. For the purposes of this paper, however, we only need to be aware of the relationship between characteristics and antecedent conditions.

${ }^{18}$ This was first proved for a special case by Augustin Cauchy in 1842 and in full generality by Sophie Kowalewski in 1875. The proof requires also that equation (2) be analytic and regular, though these further restrictions are not important for our discussion here.
} 
can be any two-dimensional open surface which passes through the vertices of the cones, whose curvature is such that it never touches the cones. A (hyper)surface on which Cauchy data is defined is known as a Cauchy surface. ${ }^{19}$

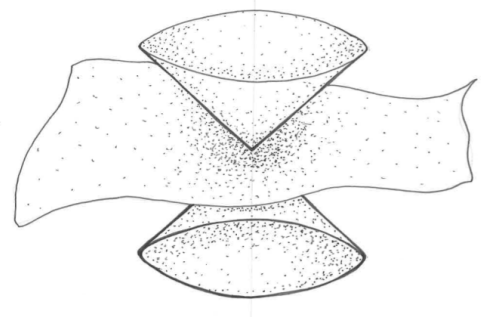

Figure 2: Conoid characteristic surfaces in $\mathbb{R}^{3}$, with a Cauchy surface. The Cauchy surface extends infinitely in all directions, and the characteristics are defined at every point on the Cauchy surface.

A linear hyperbolic PDE that satisfies criteria $W P_{1}$ and $W P_{2}$, in regions of its domain where solutions are defined for correctly formulated Cauchy data, will also satisfy $W P_{3} \cdot{ }^{20}$ This is the case, provided that solutions do not intersect and are not tangent anywhere to a characteristic, and that they do not intersect one another. ${ }^{21}$ This means that the solution function to a hyperbolic PDE describes a set of trajectories, each of which passes through a point on the Cauchy surface and propagates inside but not outside the characteristic cone defined at that point.

A canonical example of a hyperbolic equation, which has all of the geometric properties described above, is the wave equation:

$$
\sum_{i}^{m-1} \frac{\partial^{2} u}{\partial x_{i}{ }^{2}}-\frac{1}{c^{2}} \frac{\partial^{2} u}{\partial x_{m}{ }^{2}}=f\left(x_{1}, \ldots, x_{m}\right)
$$

for constant $c .^{22}$ This equation informs about propagation of electromagnetic or sound waves

\footnotetext{
${ }^{19}$ For the sake of simplicity, this paper ignores many of the issues surrounding the lack of generality of the result of the Cauchy-Kowalewski theorem about the relationship between characteristics and antecedent data. Most significantly, there well-known approaches to general relativity that involve null (characteristic) antecedent data.

${ }^{20}$ Cauchy problems which satisfy $W P_{1}$ and $W P_{2}$ but violate $W P_{3}$ are called ill-posed, and are invariably not of the hyperbolic form. They are relatively rare in physical contexts. An example is the inverse gravimetric problem. See for instance I. Rubinstein and L. Rubinstein 1998.

${ }^{21}$ There are less clear results for parabolic PDEs, but these are not the focus of the present work.

${ }^{22}$ Regarding the status of the wave equation (and other PDEs) as laws: the wave equation is derived from Hooke's law, to use conventional terminology. However, what counts as a law and what doesn't is not our concern here.
} 
in directions which we have independent reasons to regard as temporal. We can see that the second derivative of one of the independent variables, $x_{m}$, is distinguished by its negative sign. In applications, $x_{m}$ is chosen to represent time, because this equation is used to describe dynamics, i.e. time-evolution.

To summarise the results of this section, we require a clear asymmetry between the hypersurface on which antecedent data is defined and the directions in which solutions propagate, in order to define a well posed Cauchy problem. In physical applications, this means that Cauchy problems provide the most natural means for modelling time evolution, provided the dynamics of the target system are relatively simple and 'well behaved'. For this reason, the Cauchy problem has become known as the Initial Value Problem (IVP). So, Cauchy problems are informative in directions that are naturally interpreted as timelike. This fact underwrites Callender's formal argument for his TIP. However, the Cauchy problem is not the only type of problem of physical relevance. This will be emphasised by the examples given in the following two subsections. Their abilities to inform about the manifold of empirical events, considered neutrally, will be compared in section 4 .

\subsection{Informative, Non-temporal Directions}

This subsection presents a case where laws inform in non-temporal directions. Dirichlet and Neumann problems are two different sorts of BVP that can be set for second order linear PDEs of the form (1) or its linearized form (2). Dirichlet and Neumann boundary conditions are not of the correct form to ensure the existence of unique solutions for hyperbolic equations, but they are for parabolic and elliptic equations. PDEs of the parabolic type, like hyperbolic equations, inform in timelike directions, so we do not discuss them here. Elliptic PDEs, on the other hand, do not single out a privileged set of directions, despite providing powerful algorithms which are informative in many areas of physics. For correctly formed antecedent data, they satisfy criteria $W P_{1}-W P_{3}$ (despite these being originally explicated in the context of Cauchy problems), thus meeting Callender's standard of informativeness. 
A canonical example of an elliptic equation is the Poisson equation:

$$
\sum_{i} \frac{\partial^{2} u}{\partial x_{i}{ }^{2}}=f\left(x_{1}, \ldots, x_{m}\right) .
$$

This equation has many physical applications, for example for finding the electric potential for a given charge distribution. ${ }^{23}$ The form of this equation makes no distinction between spatial and temporal variables. Recall from the previous subsection that elliptic equations have no real characteristics, so, as long as we are working in real space, the Cauchy-Kowalewski theorem imposes no restrictions on where to define the antecedent data to ensure the existence of a unique solution. The form of the antecedent data, however, is important. As mentioned, Cauchy data is either overly restrictive or leads to instabilities for elliptic PDEs. Instead, we require either Dirichlet or Neumann boundary conditions defined on a closed boundary surrounding the region of interest.

A Dirichlet boundary condition takes the following form:

$B C_{D}: u\left(x_{1}, \ldots, x_{m}\right)=u_{0}\left(x_{1}, \ldots, x_{m}\right)$,

for $x_{i} \in \delta \Omega$, where $\delta \Omega$ is a closed surface or hyper-surface. In other words, a Dirichlet boundary condition gives the value of the solution on a closed boundary.

A Neumann boundary condition takes the following form:

$B C_{N}: v\left(x_{1}, \ldots, x_{m}\right) \frac{\partial u}{\partial x_{i}}\left(x_{1}, \ldots, x_{m}\right)=u_{0}\left(x_{1}, \ldots, x_{m}\right)$,

again for $x_{i} \in \delta \Omega$, where $\delta \Omega$ is a closed (hyper-)surface. The function $v$ defines a tangent vector at each point on the boundary. So, a Neumann condition gives the directional derivative of the solution on a closed boundary. Usually, only one of these types of boundary condition is required to solve an elliptic PDE such as the Poisson equation. What elliptic equations do is take information (either the solution or its directional derivative) on a closed boundary and

\footnotetext{
${ }^{23}$ There are many well-known physical applications for other elliptic PDEs, for example relating to energy functionals of fields defined over space and time, but the simple example of the Poisson equation is given simply to illustrate the geometric properties of this sort of PDE.
} 
'evolve' it inwards or outwards from that boundary. An illustration of the two-dimensional case is shown in figure 3. For the three-dimensional case, imagine information on the surface of a soap bubble propagating either outwards or inwards.

Elliptic equations can generate information in multiple directions simultaneously, and there is no special in-built asymmetry between these directions and the boundary on which the antecedent data is defined. Recall that elliptic PDEs have no real characteristics and thus no restrictions on where in the domain this boundary can be, so no particular set of directions is privileged by elliptic PDEs. We therefore have no reason to regard the informative directions of an elliptic PDE as temporal. In general these will not correspond to 'time' directions as measured by clocks.

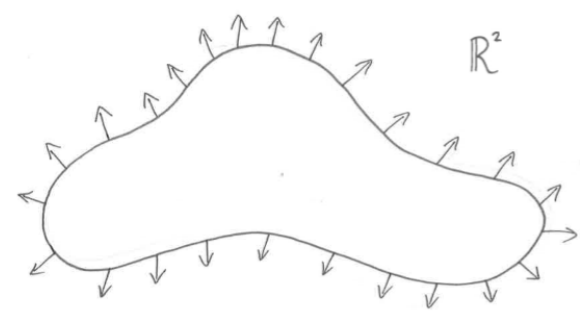

Figure 3: A closed boundary in $\mathbb{R}^{2}$ where Dirichlet or Neumann boundary conditions may be defined. The elliptic PDE then evolves this information either outwards to fill the manifold exterior to this region, or inwards to fill the interior of the boundary.

Defining time as the set of directions in which laws generate the most information leads to incompatibilities between the various 'times' emerging from different applications of elliptic PDEs, and between the 'times' arising from analyses of hyperbolic and elliptic PDEs. ${ }^{24}$ Callender's reasons for restricting attention to hyperbolic PDEs, rather than giving up on TIP, are discussed in section 4 . Next, we come to our second set of counter-examples to TIP, which are not acknowledged by Callender.

\subsection{Uninformative, Temporal Directions}

This subsection details a different sort of counterexample to TIP, namely chaotic dynamical systems, in which the directions that are ordinarily defined to be time are particularly

\footnotetext{
${ }^{24}$ Here, 'times' refers to the set of directions that are maximally informative in each case, the point being that, for elliptic equations, there is an absurdity in defining time in this way, since it does not correlate with any other more familiar definitions of time.
} 
uninformative. This type of example provides motivation for $T A P$, for the empiricist, because scientists and mathematicians working on the subject of chaos are trying to develop methods for generating information in timelike directions, despite the observed behaviour of the physical world making it especially difficult to do so.

Many physical systems are said to be unstable under perturbations of initial conditions, or chaotic, where initial conditions are now understood to be measured values of our $m-1$ independent variables, where $x_{m}=0$. This is as opposed to any of the antecedent functions $u_{0}$ and $u_{1}$ described in subsection $3.1\left(A C_{1}\right.$ and $\left.A C_{2}\right)$, or either of the $u_{0}$ s of subsection $3.2\left(B C_{D}\right.$ or $\left.B C_{N}\right) .{ }^{25}$ The models now under consideration are more directly related to empirical phenomena than the functional equations previously discussed, because they operate at a lower level of abstraction. What is meant by instability here has to do with the way the variables $x_{1}, \ldots, x_{m}$ are related according to some function $u$, which may be (but is not necessarily) the solution to a Cauchy problem. If the measured values of $x_{1}, \ldots, x_{m-1}$ at $x_{m}=0$ are perturbed slightly, giving say $\tilde{x}_{1}, \ldots, \tilde{x}_{m-1}$, then the values of these variables at $x_{m} \neq 0$ will also differ from one another, giving rise to two different functions $u=u\left(x_{1}, \ldots, x_{m-1}, x_{m}\right)$ and $\tilde{u}=u\left(\tilde{x}_{1}, \ldots, \tilde{x}_{m-1}, x_{m}\right)$. For a dynamical system, these functions will map onto two trajectories in an $m$-1-dimensional phase space, where each point on a trajectory represents the full state of the system at some value of $x_{m}$.

A dynamical system is chaotic if it is both deterministic, in the sense defined in subsection 3.1, and exhibits sensitive dependence on initial conditions (SDIC). To exhibit SDIC is to be unstable, in the sense that functions $u$ and $\tilde{u}$ will separate at an exponential rate as $x_{m}$ varies uniformly. For this definition to make sense, we let $x_{m}$ be identical in both functions, allowing it to naturally represent a time parameter. This means that the distance between phase space trajectories arising from a function, $u$, will grow exponentially with time. In practical applications, this leads to extreme inaccuracies in predictions of measurement outcomes, since the dynamical equation amplifies errors in the initially measured data. The function, $u$,

\footnotetext{
${ }^{25}$ Technically speaking, Cauchy, Dirichlet or Neumann problems whose solutions depend sensitively on the antecedent conditions defined in the previous two subsections would also count as chaotic, but this paper aims to focus on the more empirically salient cases concerned with physical measurement.
} 
therefore does not provide an empirically adequate description of the physical world outside of a very restricted range of values of $x_{m}$ close to $x_{m}=0$. That is, predictions about the future and retrodictions about the past based on these models are practically impossible. In these cases, the asymmetry between the variables $x_{1}, \ldots, x_{m-1}$ and $x_{m}$, corresponding to space and time variables respectively, arises from an asymmetry between what we have empirical access to and what we do not. It is not the case that the direction of increasing $x_{m}$ is informative; in fact, quite the opposite is the case.

There are many examples of chaotic system to choose from to illustrate this point. Unlike the linear PDEs we have been analysing so far, which can be grouped into classes which share quantitative features, models of chaotic systems are rather heterogeneous in their mathematical form. What they share is the qualitative feature of a combination of determinism and SDIC. ${ }^{26}$ The model presented here is one among many, chosen for its simplicity and physical applicability. Its qualitative features, shared by other chaotic models/systems, mean that the temporal dimension is particularly uninformative. ${ }^{27}$

The Lorenz model is a simplification of a model of convection in fluids. ${ }^{28}$ More specific details of this model than what is presented below are largely irrelevant; it must be acknowledged only that the model (and others exhibiting SDIC) were empirically motivated, and solutions can be reproduced experimentally. Other physical systems whose dynamics exhibit SDIC (but which are not modelled by the Lorenz equations) include many problems in celestial mechanics, acoustics, hydrodynamics, some quantum scenarios and even quantum gravity (see for example Diacu and Holmes 1996, Ott 1993, Palis 1993 and authors 1987 for classical cases, and Dittrich et al. 2017 for the case of quantum gravity).

\footnotetext{
${ }^{26}$ For a comprehensive analysis of the various types of model which get called 'chaotic', see Zuchowski 2017. Her work includes discussion of problems with defining chaos, and with trying to ascertain whether SDIC is a feature of the model or the system. These more involved issues are not our concern here - we assume that SDIC can arise in deterministic models (which are deterministic in the sense defined in section 3.1), and that it is a feature of the target system as well as the model because it has empirical consequences.

${ }^{27}$ The philosophical literature on chaos for the most part focuses on modelling problems, especially in 'special sciences', which are tangential to the concerns of the present work. See for instance Frigg et al. 2014, Zuchowski 2017 and Werndl 2009.

${ }^{28}$ For discussion of the physical applicability of the model, see Lücke 1976 for example.
} 
This model is a well-known example of a simple chaotic model. It is a system of ordinary differential equations as follows: ${ }^{29}$

$$
\begin{gathered}
\frac{d x}{d \tau}=-\sigma x+\sigma y, \\
\frac{d y}{d \tau}=-x z+r x-y, \\
\frac{d z}{d \tau}=x y-b z
\end{gathered}
$$

where $x$ is proportional to the intensity of convective motion, $y$ is proportional to the temperature difference between ascending and descending currents, and $z$ is proportional to the deviation of vertical temperature from linearity; $\sigma, r$ and $b$ are constants; and $\tau$ is a time parameter. To connect to our earlier notation, let $x_{1}=x, x_{2}=y, x_{3}=z$ and $x_{4}=\tau$. We are now playing a different game to Callender. Rather than identifying the most informative directions and calling them 'time', we are looking at physical equations with a time parameter already defined in order to investigate its properties. Equations (6) to (8) each depend on instantaneous values of $x, y$ and $z$, and solutions give trajectories describing how these variables change over time. Initial conditions for this system are just values of the three variables at $\tau=0$.

Depending on the values of the constants $\sigma, r$ and $b$, and depending on the initial values of $x, y$ and $z$, solutions of this system can vary dramatically as a result of slight perturbations of initial data. This phenomenon can be quantified by calculating Lyapunov exponents, which measure the rate of divergence between neighbouring trajectories. To spell this out, the solutions $u(x, y, z, \tau)$ and $u(\tilde{x}, \tilde{y}, \tilde{z}, \tau)$ will diverge at a rate of $e^{\lambda \tau}$, where $\lambda>0$ is known as the Lyuponov exponent. ${ }^{30}$ An illustration of this phenomenon is shown in figure 4.

Mathematically, this is a consequence of the nonlinear terms in equations (7) and (8). ${ }^{31}$ For

\footnotetext{
${ }^{29}$ An ordinary differential equation can be thought of as a special case of a partial differential equation, to connect this to our earlier discussion of PDEs. Many chaotic models do consist of systems of PDEs but these tend to be much more complicated; to present such an example here would distract from our main discussion.

${ }^{30}$ Calculating Lyuponov exponents is just one widely used method for quantifying SDIC which is appropriate in many contexts of physical interest, though not all.

${ }^{31}$ More generally, chaos can arise in deterministic systems either as the result of non-linearity, or of discontinuity in the sense of violations of the condition $W P_{3}$ defined in subsection 3.1. This second variety of chaos is much
} 


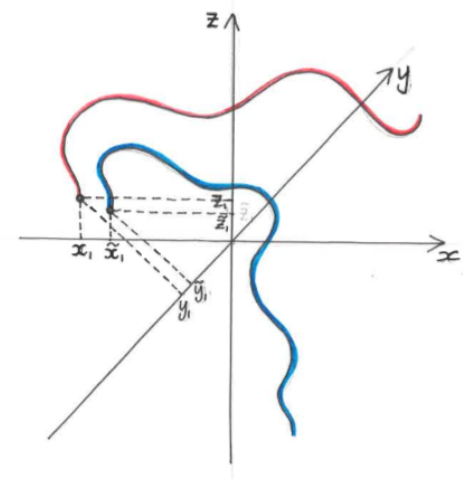

Figure 4: A pair of chaotic trajectories in threedimensional phase space. $u(x, y, z, 0)$ is depicted in red and $u(\tilde{x}, \tilde{y}, \tilde{z}, 0)$ in blue. In practice, the two sets of initial values, $\left(x_{1}, y_{1}, z_{1}\right)$ and $\left(\tilde{x}_{1}, \tilde{y}_{1}, \tilde{z}_{1}\right)$ may be arbitrarily close to one another.

this reason, despite the equations having been designed to inform in timelike directions, the information generated can be highly unstable. It may therefore be completely inaccurate with respect to the empirical world because we never have access to arbitrarily precise data through measurement. Approximate predictions about empirical phenomena are generated through statistical analysis of many solutions. This kind of analysis often involves identifying which sets of initial data lead to stable solutions and which do not, rather than simply providing information about what will be measured given one particular set of initial conditions. The empirically adequate information that one can glean from a model of a chaotic system therefore does not correspond to any direction or set of directions on the manifold.

The next section explains in more detail why the above cases are counterexamples to TIP, and why they suggest an alternative proposal, $T A P$.

\section{Comparison}

This section compares the informativeness (in Callender's sense) of the models described: hyperbolic PDEs are compared with elliptic PDEs in subsection 4.1, and with chaotic systems in 4.2. In subsection 4.3, the positive proposal of this paper, $T A P$, is reiterated and compared with the reading of Callender's argument put forward in Baron and Evans forthcoming. Both are forms of perspectivalism, and can be made compatible with one another in the present case.

less studied than the non-linear cases, and has been shown only to arise in systems with infinite-dimensional state space. See Kalmar-Nagy and Kiss 2017 for an example. 
Callender's argument for TIP relies on his focus on hyperbolic PDEs which support well posed Cauchy problems. Once we restrict attention to PDEs of this form, as shown in section 3.1 , the set of directions that they inform over do indeed correspond to timelike directions in physical applications. It is interesting to observe how their geometric structure enables them to effectively inform over timelike directions, in their domains of applicability. Does this give us any reason to posit a general connection between time and informative strength in physical laws? To answer positively to this question, as Callender does, requires some justification for restricting attention to hyperbolic PDEs, and for taking them to be representative of physical laws in general. Callender justifies this move by appealing to the theoretical virtue of strength as informativeness, inspired by the BSA.

Recall that Callender stipulates that the 'degree to which a theory is informative is determined by how much of the world it manages to imply' (Callender 2017, p.143). His claim is then that hyperbolic PDEs with Cauchy data provide laws that are maximally informative in this sense. Is this claim correct? The answer to this question depends on what we consider 'the world' to refer to. Taking our empiricist principles seriously, even in a minimal sense, requires that what we mean by 'the world' should make some contact with empirically accessed or accessible phenomena.

\subsection{Hyperbolic vs Elliptic PDEs}

Callender uses the three criteria (labelled $W P_{1}, W P_{2}$ and $W P_{3}$ in section 3.1) as a way of making his notion of informativeness precise. Although Jacques Hadamard first outlined these conditions in his work on the Cauchy problem (see Hadamard 2003, p.40; originally published in 1923), it is not the case that only well posed Cauchy problems meet them. They are sufficient conditions for a Cauchy problem to be 'well posed', but it is not the case that any problem which meets them is a well posed Cauchy problem. A Cauchy problem is defined by the type of antecedent data prescribed for it, and the three conditions were added in order to distinguish those Cauchy problems that are 'well posed' from those that are not. 
What Callender has overlooked is the fact that elliptic PDEs such as the Poisson equation, together with the correct sort of antecedent data such as Dirichlet or Neumann boundary conditions, also meet conditions $W P_{1}, W P_{2}$ and $W P_{3}$. That is, if such a problem is correctly formulated, there exist unique solutions for each set of antecedent data which vary continuously with that data. What's more, elliptic PDEs in general require only one boundary condition for their solution, in contrast to hyperbolic PDEs which require two. Considered in purely formal terms, then, elliptic PDEs are at least as informative as their hyperbolic cousins. That is, their ability to inform is at least equivalent, provided we make no assumptions regarding the nature of the systems we wish to be informed about.

Dirichlet or Neumann boundary value data is as empirically accessible as Cauchy data. ${ }^{32}$ There is a sense in which all three kinds of data are mathematical idealisations, and none of them are directly empirically accessible. Imagine for a moment the practical impossibility of making empirical measurements of some physical quantity on a Cauchy surface. Cauchy surfaces are spatially unbounded and so to collect a complete set of Cauchy data would require an infinite number of measurements. Measurement is also itself a physical process, meaning that data takes time to gather and we do not ever have empirical access to every point on a Cauchy surface simultaneously. The idea of an 'initial' function defined on a Cauchy surface, and the stark distinction between time and space built into the definition of this sort of problem, is part of the idealisation. In practice, symmetry principles are used so that the initial function can be stipulated or found using an elliptic equation, as mentioned in subsection 3.2. It makes little sense to compare the availability of initial and boundary data, since both involve a high degree of idealisation as well as empirical measurement. It also makes little sense to compare how often each kind of problem appears in physical applications. ${ }^{33}$ Both kinds of problem appear in physics, both are used, and both bear some relation to describing and predicting patterns in empirical data.

\footnotetext{
${ }^{32}$ I would like to thank an anonymous referee for raising the issue of comparing the empirical accessibility of the different kinds of data.

${ }^{33}$ It could be claimed, for example, that hyperbolic PDEs are more 'typical' in physical applications. It is not clear how typicality should be judged in the present case: as typicality of use or of physical processes. In either case, there is no measure over the space of PDEs in science, and so this sort of claim would require further evidence if it is to be endorsed. I would like to thank an anonymous referee for raising the issue of typicality.
} 
This point is not entirely ignored by Callender. Although he does not explicitly compare their informativeness, he does briefly argue for the fundamentality of hyperbolic systems, mentioning that 'Geroch (1996) writes, "elliptic and parabolic systems arise in all cases as mere approximations of hyperbolic systems"'. He goes on to say that this point is 'sometimes demonstrably correct: many equations not of [hyperbolic] form (8.1) can be derived from ones that are by taking limits or making simplifying assumptions. The elliptic Poisson equation, for instance, is a truncation of the linear hyperbolic Maxwell equations' (Callender 2017, p.165; emphasis added). There arises here the obvious objection that a point which is only sometimes demonstrably correct cannot be a suitable candidate for motivating a general claim, especially given that the 'sometimes' in fact refers only to scalar theories. Gauge theories, including general relativity, have constraint equations built in, in the form of elliptic equations, which must be solved in order to set the Cauchy problem.

The above point also relies on taking fundamentality to mean 'non-approximate', when in fact there is no consensus in the literature about what this term means, even in specific contexts. ${ }^{34}$ We are then faced with at least two options for how to read 'non-approximate'. Callender's analysis of the above quotation from Geroch suggests a formal reading, where approximation is a particular kind of mathematical derivation. This reading is antagonistic to our empiricist principles, because no mention is made of fit to empirical data. Hyperbolic systems are not more exact (accurate and/or precise) than elliptic systems in the sense of being closer approximations to what is measured in the physical world. ${ }^{35}$ Callender's reasoning is thus not sufficient to favour the Maxwell equations over the Poisson equation, or to prefer hyperbolic over elliptic systems in general. No argument is given for regarding hyperbolic systems as more informative than elliptic systems. Neither can be considered in isolation as the best guide to investigating the role played by time in physical laws. They are simply used in different ways,

\footnotetext{
${ }^{34}$ It is not the intention here to engage in discussion about what exactly is meant by 'fundamental'. The interested reader may refer to, for example, Tahko 2018 and references therein, and especially Crowther 2019 for the the case of physical theories. The issue is mentioned here only for the sake of completeness, and readers may have in mind some basic intuitive notions of fundamentality.

${ }^{35}$ This point also applies to chaotic systems, and will be returned to in subsection 4.2.
} 
depending on the kind of information sought.

When comparing the informativeness of hyperbolic and elliptic systems, it is important to be aware of what it is we wish to be informed about. That is, our aims must be acknowledged. If we wish to be informed about dynamics, an elliptic system will not do. Given that the study of dynamics just is the study of processes evolving in time, it is to be expected that the mathematical tools designed for this purpose distinguish timelike directions, as hyperbolic PDEs do.

\subsection{Stable vs Chaotic Dynamical Systems}

Chaos is studied as a branch of dynamical systems theory, where the aim is to inform in temporal directions. Chaotic dynamical systems are characterised by the fact that this task is made particularly difficult by the behaviour of such systems. The point is not to dwell on the metaphysics of laws, but simply to apply the principles of the BSA. This means taking some set of physical phenomena and looking for the best (strongest and simplest) methods for describing the observed regularities and for making empirically relevant predictions.

As emphasised in section 3.3, the study of chaotic systems has empirical motivations. The goal is to develop the most effective methods for making empirical predictions in timelike directions, a procedure which often involves a variety of techniques to mitigate the instability. In general it is the case with these systems that reasonably accurate predictions can be made only for short timescales, even when using our best predictive tools. Our best description of such a system should surely take this into account. So, if we seek the best description of some physical system exhibiting chaotic behaviour, we see quite the opposite of Callender's TIP. That is, the temporal directions about which we wish to be informed are especially uninformative.

The informativeness of hyperbolic PDE systems depends on the behaviour of the target system - a hyperbolic system does not always faithfully encode the observed dynamics. Temporal directions in well posed Cauchy problems have the potential to be especially informative 
due to the way the problems have been designed. However, they inform only about possible (non-actual) empirical phenomena unless the dynamics of the target system are 'well behaved' in the sense of being non-chaotic. In other cases, alternative methods (usually statistical) are used to try to make the best possible empirical predictions, but it does not make sense to speak of 'the direction(s) of information propagation' when such methods are employed. The dynamical equations generate information over time, but this information is wildly inaccurate when compared to empirical phenomena. The dynamical equations for such systems are also not at all simple, usually being characterised by non-linearity. Statistical analysis of phase portraits is more informative in the sense of giving accurate information, but the information generated is not temporally oriented. The non-temporally-oriented statistical methods also keep track of the regularities of chaotic systems, but these are generally 'higher level' or 'emergent' regularities. ${ }^{36}$ The point is not exactly to decide which formal methods count as laws, either 'properly speaking' or according to the BSA, but to understand how we come to be informed about physical systems and the role time plays.

Callender recognises that 'there is no guarantee that the best theory will contain algorithms that permit such a nice time', but claims that 'nature must be kind. So far, it has been.' (Callender 2017, p.145). Chaotic phenomena shows that this claim is false: they are cases where nature is not 'kind', where the best theories consist of amalgamations of statistical methods which do not contain algorithms permitting a 'nice time'. Information in temporal directions is what is sought, but accurate and precise information (in the sense of correctly corresponding to empirical data) is not what is found. The existence of systems exhibiting such behaviour in the physical world, and recognition of the great efforts made by mathematical physicists to generate at least approximate short-term predictions in such cases, together motivate replacing TIP with TAP.

\footnotetext{
${ }^{36}$ This is mentioned in relation to a point raised by an anonymous referee, who questioned whether the formal methods presented count as laws. Laws, for the Humean, simply keep track of empirical regularities. For the best systems theorist, they do so in the 'best' way. If the 'best' way of describing a chaotic system is not strong or simple enough to satisfy certain best systems theorists, they must admit that there are empirical phenomena which exhibit some regularity but about which there are no laws.
} 
Objections to my argument from chaos may again appeal to the supposed fundamentality of hyperbolic systems, as well as the non-fundamentality of chaotic systems. This objection would provide an alternative reason for favouring hyperbolic over chaotic systems, despite their informativeness about the empirical world being incomparable, thus supporting a weaker version of TIP on the grounds that the informative directions in our most fundamental laws are timelike. Taking 'fundamental' to mean either 'non-approximate' or 'exact', so long as we are empiricist and exactness has something to do with fit to empirical data, means that it is simply not the case that hyperbolic systems are more fundamental than chaotic ones.

If chaos were to arise only in complex systems where approximations to the underlying dynamics are made, the objection to TAP from fundamentality, where 'fundamental' roughly means 'exact' or 'non-approximate', might carry some force. However, there is no entailment in either direction between chaos and complexity, and chaos often arises in systems which are not complex. ${ }^{37}$ The approximate statistical methods mentioned in subsection 3.3 are used in the study of chaotic dynamical systems to deal with the SDIC that is present in the more precise description. SDIC does not arise as a result of approximation. This refutes the possible objection to the significance of chaotic systems based on their non-fundamentality, where 'fundamental' is taken to mean 'non-approximate'.

If 'fundamental' were instead taken to refer to that which is described by putatively fundamental theories, such as general relativity, quantum field theory or quantum gravity, the objection that chaotic dynamics are non-fundamental still does not hold. As maintained in (Dittrich et al. 2017), ' full general relativity is almost certainly chaotic'. The same authors then go on to make the case that quantum gravity involves chaotic dynamics. Of course these claims are controversial. The idea that general relativity should involve chaos in the form of non-linear equations has met with some resistance, perhaps based largely on aesthetic concerns and stipulations of physical reasonableness. However, as John Wheeler pointed out, 'it is no objection to the physical reasonableness of general relativity to find that the equations

\footnotetext{
${ }^{37}$ See for instance Ladyman, Lambert, and Wiesner 2013 for discussion of where chaos and complexity come apart.
} 
are non-linear. To argue that physics "does not like" non-linear equations is as futile as standing under the roar of Niagra Falls and trying to reason away the hard reality of non-linear hydrodynamics.' (Wheeler 1964). Regarding the quantum case, it is an established fact that chaotic dynamics occur in quantum systems (see for example Haake 1991 and Stöckmann 2000 for comprehensive treatments of the subject, with reference to experimental results), although here the situation is not nearly as straightforward as in the classical case described in section 3.3.

The discussion of fundamentality is somewhat tangential to our main discussion. Empiricist principles commit us to take seriously the available empirical data, and this alone does not involve prioritising certain sets of data over others. The issue of fundamentality is mentioned only to alleviate the potential concerns of those empiricists who are also reductionists, who believe we should not be looking to 'special sciences' to address metaphysical issues. Chaos is not only a 'special science' phenomenon, and so should be taken into consideration in a thorough investigation into the role played by time in physical theories. What this phenomenon demonstrates is a failure of our ability to predict, despite scientists' best efforts. This failure is enforced by the dynamical behaviour of the physical world. It shows that it is neither physics in general, nor the behaviour of the physical world, that grants time its informative strength in those special cases where temporal directions in laws successfully inform. Rather, it is the way in which scientists construct laws, to provide the kind of information that is sought, which is very often information about unknown, empirically inaccessible temporal directions.

\subsection{Two Forms of Perspectivalism}

The arguments given in this paper have been in support of the claim, referred to as $T A P$, that the connection Callender makes between time and informative strength in physical laws holds only relative to a particular set of aims and research interests. This was demonstrated by the counterexamples presented. TAP can be viewed as a perspectival reading of one of the central claims of Callender's proposal, which I have been referring to as TIP. The 'perspective' to which it is reduced is understood to mean some collection of research perspectives. It is suggested that physics which aims to make predictions in directions that are already understood 
to be 'time' will have 'time' as the informative direction in its laws. When such laws do not generate accurate and precise information due to the observed behaviour of the systems to which they are applied, as is the case for chaotic systems, alternative techniques are used to gain as much information as possible in 'time' directions.

The proposal put forward in Baron and Evans forthcoming is a different sort of persepctival reading, where 'perspective' is understood as the epistemic perspectives of some group of agents, in this case the human species, or 'creatures like us'. Their 'temporal perspectivalism' is explicitly based on the 'causal perspectivalism' of Huw Price (Price 2005). To explain what is meant by a perspectival claim, Price uses the example of foreigners, reminding his readers that who is regarded as 'foreign' depends on perspective. The people who are 'foreign' to Frenchmen are different to those who are 'foreign' to Englishmen, but this does not mean that either party is incorrect about their judgements. The same kind of analogy can be applied to the ways in which physical laws are used to generate information. There will be 'no-fault' disagreements about which directions are informative, depending on what kind of information is sought. There will also be potential 'no-fault' disagreements about which directions are informative depending on the epistemic perspective, a hypothesis which can be fleshed out more thoroughly by identifying what it is about a particular epistemic perspective which makes it suitable for gathering information along a particular set of directions. The former, interest-relative, perspectivalism is what has been referred to here as $T A P$. The latter is the species-relative perspectivalism developed in Baron and Evans forthcoming, is here applied to 'informative directions' rather than 'time directions' as in the original work. The two forms of perspectivalism are compatible, when applied to the same claim, since epistemic perspectives and research perspectives identified by aims and interests are not independent. 


\section{Conclusion}

Callender's claim $(T I P)$ is that our most informative models inform in timelike directions. This paper has proposed a modification to this claim $(T A P)$, which says instead that what is the most informative set of directions in the most informative model depends on what we wish to be informed about, that is, on our aims. The extent to which models geometrically distinguish time from space depends on the extent to which we wish to be informed about processes evolving in time (dynamics), where we have a host of independent reasons to regard this evolution as happening in time. This point is highlighted by consideration of elliptic systems, where the aim is to find information in spatial directions and not to study dynamics.

The extent to which the 'time' directions in a model, designed with the aim of informing over time, are in fact informative with respect to a physical system of interest depends on the behaviour of that system. This is demonstrated by the case of chaotic systems, where the aim is to study dynamics but the 'time' directions in the models are uninformative. The empiricist principles Callender set out with are particularly important with respect to this last point, because they force us to think about informativeness in terms of correspondence of a theory or law with empirical data. The proposal, $T A P$, should be understood as a fairly weak claim: scientists often (but not always) wish to generate information in temporal directions. Where the aim is to generate such information, and where the physical world behaves in such a way as to allow for such information to be generated (both are required), strong algorithms may be applied which, unsurprisingly, inform in temporal directions.

These messages illustrate the more general problem that the actual achievements or capabilities of science can sometimes fall short of its aims. Where some scientific discipline sets out to make accurate and precise empirical predictions, there always exists the possibility of failure. In the case of chaotic systems, it remains to be seen whether this is only a failure of our current best models to achieve the predictive aims, or an in principle incapability of any model to make the kinds of predictions sought. The way that the subject has been presented here suggests the latter interpretation, although this will most likely depend on the particular 
system in question. Future developments in the field may also turn out to demonstrate otherwise.

To return very briefly to the question of a philosophical underpinning for the various asymmetries between time and space, Callender's account cannot succeed. This paper has pointed out the lack of generality of the key component of his account, namely the connection between time and informative strength in physical laws, and suggested a perspectival reading of it. Recognising that this claim cannot underpin the asymmetries between time and space, its metaphysical thrust consists in a relationship between the world and information-gathering systems such as ourselves. This paper has pointed out that this relation only sometimes obtains, but that often it is the objective of scientific inquiry to forge such a relation. Identifying when this relation obtains and when it doesn't, and asking why it does or does not obtain in these cases, would be interesting projects for deepening the analysis. 


\section{References}

[1] Various authors. Dynamical Chaos. The Royal Society, 1987.

[2] Sam Baron and Peter W. Evans. "What's So Spatial About Time Anyway?" In: British Journal for the Philosophy of Science (forthcoming). DOI: 10.1093/bjps/axy077.

[3] Gordon Belot. Geometric possibility. Oxford University Press, 2011.

[4] Karen Bennett and Brian McLaughlin. "Supervenience". In: Stanford Encyclopedia of Philosophy (2005).

[5] Craig Callender. What makes time special? Oxford University Press, 2017.

[6] Rudolf Carnap. "Empiricism, semantics, and ontology". In: The philosophy of science (1991), pp. 85-98.

[7] Jonathan Cohen and Craig Callender. "A better best system account of lawhood". In: Philosophical Studies 145.1 (2009), pp. 1-34.

[8] John Cottingham. The rationalists. Oxford University Press, 1988.

[9] Karen Crowther. "When do we stop digging? Conditions on a fundamental theory of physics". In: What is Fundamental? Springer, 2019, pp. 123-133.

[10] Florin Diacu and Philip Holmes. Celestial Encounters. Princeton University Press, 1996.

[11] Bianca Dittrich et al. "Can chaos be observed in quantum gravity?" In: Physics Letters B 769 (2017), pp. 554-560.

[12] Paul Feyerabend. "Problems of empiricism". In: Philosophical Papers (1965).

[13] Roman Frigg et al. "Laplace's demon and the adventures of his apprentices". In: Philosophy of Science 81.1 (2014), pp. 31-59.

[14] Paul Garabedian. Partial Differential Equations. John Wiley Sons, inc., 1964.

[15] Ronald N Giere. Scientific perspectivism. University of Chicago Press, 2010.

[16] Anil Gupta. Empiricism and Experience. Oxford University Press, 2006.

[17] Fritz Haake. "Quantum signatures of chaos". In: Quantum Coherence in Mesoscopic Systems. Springer, 1991, pp. 583-595. 
[18] Jacques Hadamard. Lectures on Cauchy's problem in linear partial differential equations. Courier Corporation, 2003.

[19] Jenann Ismael. "How do causes depend on us? The many faces of perspectivalism". In: Synthese 193.1 (2016), pp. 245-267.

[20] Tamas Kalmar-Nagy and Marton Kiss. "Complexity in Linear Systems: A Chaotic Linear Operator on the Space of Odd-Periodic Functions". In: Complexity 2017 (2017).

[21] Sergiu Klainerman. "PDE as a unified subject". In: Visions in Mathematics. Springer, 2010, pp. 279-315.

[22] James Ladyman, James Lambert, and Karoline Wiesner. "What is a complex system?" In: European Journal for Philosophy of Science 3.1 (2013), pp. 33-67.

[23] Baptiste Le Bihan and Niels Linnemann. "Have we lost spacetime on the way? Narrowing the gap between general relativity and quantum gravity". In: Studies in History and Philosophy of Science Part B: Studies in History and Philosophy of Modern Physics 65 (2019), pp. 112-121.

[24] David Lewis. "New work for a theory of universals". In: Australasian journal of philosophy 61.4 (1983), pp. 343-377.

[25] David Lewis. Papers in metaphysics and epistemology. Vol. 2. Cambridge University Press, 1999.

[26] Barry Loewer. "Humean supervenience". In: Philosophical Topics 24.1 (1996), pp. 101127.

[27] M Lücke. "Statistical dynamics of the Lorenz model". In: Journal of Statistical Physics 15.6 (1976), pp. 455-475.

[28] Michela Massimi. "A Perspectivalist Better Best System Account of Lawhood". In: W. Ott and L. Patton (2018), pp. 139-57.

[29] Michela Massimi. "Bringing real realism back home: A perspectival slant". In: The Philosophy of Philip Kitcher (2016). 
[30] Michela Massimi. "Four kinds of perspectival truth". In: Philosophy and Phenomenological Research 96.2 (2018), pp. 342-359.

[31] Tim Maudlin. "Why Be Humean?" In: The metaphysics within physics (2007), pp. 50-77.

[32] Edward Ott. Chaos in Dynamical Systems. Cambridge University Press, 1993.

[33] F. Palis J.; Takens. Hyperbolicity Sensitive Chaotic Dynamics at Homoclinic Bifurcations. Cambridge University Press, 1993.

[34] Huw Price. "Causal perspectivalism". In: (2005).

[35] Huw Price and Richard Corry. Causation, physics, and the constitution of reality: Russell's republic revisited. Oxford University Press on Demand, 2007.

[36] Willard V Quine. "Main trends in recent philosophy: Two dogmas of empiricism". In: The philosophical review (1951), pp. 20-43.

[37] Clark Robinson. Dynamical systems: stability, symbolic dynamics, and chaos. CRC press, 1998.

[38] Isaak Rubinstein and Lev Rubinstein. Partial Differential Equations in Classical Mathematical Physics. Cambridge University Press, 1998.

[39] Hans-Jürgen Stöckmann. Quantum chaos: an introduction. AAPT, 2000.

[40] Tuomas E. Tahko. "Fundamentality". In: The Stanford Encyclopedia of Philosophy. Ed. by Edward N. Zalta. Fall 2018. Metaphysics Research Lab, Stanford University, 2018.

[41] Paul Teller. "Two models of truth". In: Analysis 71.3 (2011), pp. 465-472.

[42] Charlotte Werndl. "What are the new implications of chaos for unpredictability?" In: The British Journal for the Philosophy of Science 60.1 (2009), pp. 195-220.

[43] John Archibald Wheeler. "Geometrodynamics and the issue of final state". In: Relativity, Groupos and Topology eds DeWitt CM and DeWitt BS (1964).

[44] Lena C Zuchowski. A philosophical analysis of chaos theory. Springer, 2017.

references.bib 


\section{References}

References @bookcallender2017makes, title=What makes time special?, author=Callender, Craig, year=2017, publisher=Oxford University Press @articlelewis1983new, title=New work for a theory of universals, author=Lewis, David, journal=Australasian journal of philosophy, volume $=61, \quad$ number $=4, \quad$ pages $=343$ 377, year=1983, publisher=Taylor \& Francis Group @booklewis1999papers, title=Papers in metaphysics and epistemology, author=Lewis, David, volume $=2$, year=1999, publisher=Cambridge University Press @articlebennett2005supervenience, title=Supervenience, author=Bennett, Karen and McLaughlin, Brian, journal=Stanford Encyclopedia of Philosophy, year=2005 @articlemassimi2018perspectivalist, title=A Perspectivalist Better Best System Account of Lawhood, author=Massimi, Michela, journal=W. Ott and L. Patton, pages=139-57, year=2018 @articlecohen2009better, title $=\mathrm{A}$ better best system account of lawhood, author=Cohen, Jonathan and Callender, Craig, journal=Philosophical Studies, volume=145, number $=1$, pages $=1-$ 34, year=2009, publisher=Springer @articlemaudlin2007humean, title=Why Be Humean?, author $=$ Maudlin, Tim, journal $=$ The metaphysics within physics, pages $=50$ 77, year=2007, publisher=Oxford University Press @articleloewer1996humean, title=Humean supervenience, author=Loewer, Barry, journal=Philosophical Topics, volume $=24$, number=1, pages=101-127, year=1996 @bookbelot2011geometric, title=Geometric possibility, author=Belot, Gordon, publisher=Oxford University Press, year=2011 @articlebaron2018s, year = forthcoming, author = Sam Baron and Peter W. Evans, journal = British Journal for the Philosophy of Science, title $=$ What's So Spatial About Time Anyway?, doi $=10.1093 /$ bjps/axy077 @articlemassimi2018four, title $=$ Four kinds of perspectival truth, author $=$ Massimi, Michela, journal=Philosophy and Phenomenological Research, volume $=96$, number $=2$, pages $=342-359$, year $=2018$, publisher=Wiley Online Library @bookcottingham1988rationalists, title=The rationalists, author=Cottingham, John, publisher=Oxford University Press, year=1988 @articlequine1951main, title=Main trends in recent philosophy: Two dogmas of empiricism, author=Quine, Willard V, journal=The philosophical review, pages $=20-43$, year=1951, 
publisher=JSTOR @articlecarnap1991empiricism, title=Empiricism, semantics, and ontology, author=Carnap, Rudolf, journal=The philosophy of science, pages $=85-98$, year=1991, publisher=The MIT Press Cambridge @articlefeyerabend1965problems, title $=$ Problems of empiricism, author=Feyerabend, Paul, journal=Philosophical Papers, year=1965 @bookhadamard2003lectures, title=Lectures on Cauchy's problem in linear partial differential equations, author $=$ Hadamard, Jacques, year $=2003$, publisher $=$ Courier Corporation @inproceedingsklainerman1983long, title=Long time behavior of solutions to nonlinear wave equations, author=Klainerman, Sergiu, booktitle=Proceedings of the International Congress of Mathematicians, Warsaw,(1983), pages $=1209-1215$, year=1983 @incollectionklainerman2010pde, title=PDE as a unified subject, author $=$ Klainerman, Sergiu, booktitle $=$ Visions in Mathematics, pages $=279-315$, year $=2010$, publisher=Springer @bookrobinson1998dynamical, title=Dynamical systems: stability, symbolic dynamics, and chaos, author $=$ Robinson, Clark, year $=1998$, publisher $=\mathrm{CRC}$ press @bookzuchowski2017philosophical, title=A philosophical analysis of chaos theory, author=Zuchowski, Lena C, year=2017, publisher=Springer @articlefrigg2014laplace, title=Laplace's demon and the adventures of his apprentices, author $=$ Frigg, Roman and Bradley, Seamus and Du, Hailiang and Smith, Leonard A, journal=Philosophy of Science, volume $=81$, number $=1$, pages $=31-59$, year $=2014$, publisher $=$ University of Chicago Press Chicago, IL @articlewerndl2009new, title=What are the new implications of chaos for unpredictability?, author=Werndl, Charlotte, journal=The British Journal for the Philosophy of Science, volume $=60$, number $=1$, pages $=195-220$, year $=2009$, publisher $=$ Oxford University Press @articlelucke1976statistical, title=Statistical dynamics of the Lorenz model, author=Lücke, M, journal=Journal of Statistical Physics, volume=15, number $=6$, pages $=455-475$, year $=1976$, publisher $=$ Springer $@$ bookgarabedian1964partial, title=Partial Differential Equations, author $=$ Garabedian, Paul, year=1964, publisher $=$ John Wiley Sons, inc. @bookdiacu1996celestial, title=Celestial Encounters, author=Diacu, Florin and Holmes, Philip, year=1996, publisher=Princeton University Press @bookrubinstein1998partial, title=Partial Differential Equations in Classical Mathematical Physics, author=Rubinstein, Isaak and Rubinstein, Lev, year=1998, publisher $=$ Cambridge 
University Press @bookott1993chaos, title=Chaos in Dynamical Systems, author=Ott, Edward, year=1993, publisher=Cambridge University Press @bookgupta2006empiricism, title=Empiricism and Experience, author $=$ Gupta, Anil, year=2006, publisher $=$ Oxford University Press@ @bookpalis1993hyperb, title=Hyperbolicity Sensitive Chaotic Dynamics at Homoclinic Bifurcations, author=Palis, J.; Takens, F., year=1993, publisher=Cambridge University Press@ @bookroyal1987dynamical, title=Dynamical Chaos, author=Various authors, year=1987, publisher=The Royal Society @articledittrich2017can, title=Can chaos be observed in quantum gravity?, author=Dittrich, Bianca and Hohn, Philipp A and Koslowski, Tim A and Nelson, Mike I, journal=Physics Letters B, volume $=769$, pages $=554-560$, year $=2017$, publisher $=$ Elsevier @ $@$ bookprice2007causation, title=Causation, physics, and the constitution of reality: Russell's republic revisited, author $=$ Price, Huw and Corry, Richard, year $=2007$, publisher $=$ Oxford University Press on Demand @articleismael2016causes, title=How do causes depend on us? The many faces of perspectivalism, author $=$ Ismael, Jenann, journal $=$ Synthese, volume $=193$, number $=1$, pages $=245-267$, year $=2016$, publisher $=$ Springer @articlemassimi2016bringing, title=Bringing real realism back home: A perspectival slant, author=Massimi, Michela, journal=The Philosophy of Philip Kitcher, year=2016, publisher=Oxford University Press Oxford @articleladyman2013complex, title=What is a complex system?, author=Ladyman, James and Lambert, James and Wiesner, Karoline, journal=European Journal for Philosophy of Science, volume=3, number $=1$, pages $=33-67$, year=2013, publisher=Springer @articlele2019have, title=Have we lost spacetime on the way? Narrowing the gap between general relativity and quantum gravity, author=Le Bihan, Baptiste and Linnemann, Niels, journal=Studies in History and Philosophy of Science Part B: Studies in History and Philosophy of Modern Physics, volume=65, pages $=112-121$, year=2019, publisher $=$ Elsevier @articlewheeler1964geometrodynamics, title $=$ Geometrodynamics and the issue of final state, author $=$ Wheeler, John Archibald, journal= Relativity, Groupos and Topology eds DeWitt CM and DeWitt BS, year=1964 @incollectionhaake1991quantum, title=Quantum signatures of chaos, author=Haake, Fritz, booktitle=Quantum Coherence in Mesoscopic Systems, pages=583-595, year=1991, 
publisher=Springer @bookstockmann2000quantum, title=Quantum chaos: an introduction, author=Stöckmann, Hans-Jürgen, year=2000, publisher=AAPT @InCollectionsepfundamentality, author = Tahko, Tuomas E., title = Fundamentality, booktitle = The Stanford Encyclopedia of Philosophy, editor = Edward N. Zalta, howpublished $=$ https://plato.stanford.edu/archives/fall2018/entries/fundamentality/, year $=2018$, edition $=$ Fall 2018, publisher $=$ Metaphysics Research Lab, Stanford University @bookgiere2010scientific, title=Scientific perspectivism, author=Giere, Ronald N, year=2010, publisher=University of Chicago Press @articleteller2011two, title=Two models of truth, author=Teller, Paul, journal=Analysis, volume $=71$, number $=3$, pages $=465-472$, year $=2011$, publisher $=$ Oxford University Press @articlekiss2017complexity, title=Complexity in Linear Systems: A Chaotic Linear Operator on the Space of Odd-Periodic Functions, author=Kalmar-Nagy, Tamas and Kiss, Marton, journal=Complexity, volume=2017, year=2017, publisher=Wiley @incollectioncrowther2019we, title=When do we stop digging? Conditions on a fundamental theory of physics, author $=$ Crowther, Karen, booktitle $=$ What is Fundamental?, pages $=123-133$, year=2019, publisher=Springer @articleprice2005causal, title=Causal perspectivalism, author $=$ Price, Huw, year $=2005$ 